\title{
The preventive function of low-dose aspirin in mice with preeclampsia induced by PPAR-y antagonist
}

\author{
Yongbing Guo \\ Peking University First Hospital \\ Yuchun Zhu \\ Peking University First Hospital \\ Yu Sun ( $D$ sunyu2491@163.com ) \\ Peking University First Hospital \\ Huixia Yang \\ Peking University First Hospital
}

\section{Research Article}

Keywords: Peroxisome proliferator activated receptor- $\gamma$, Low-dose aspirin, Preeclampsia, Dosedependent

Posted Date: October 28th, 2021

DOI: https://doi.org/10.21203/rs.3.rs-877595/v1

License: (1) (1) This work is licensed under a Creative Commons Attribution 4.0 International License. Read Full License 


\title{
The preventive function of low-dose aspirin in mice with preeclampsia induced by PPAR- $\gamma$ antagonist
}

Yongbing Guo, Yuchun Zhu, Yu Sun*, Huixia Yang

Department of Obstetrics and Gynecology, Peking University First Hospital, Beijing, PR China Yongbing Guo and Yuchun Zhu are co-first authors in this article.

\begin{abstract}
Background:In the present study, PPAR- $\gamma$ antagonist was used to induce preeclampsia model in mice to explore the intervention effect and mechanism of aspirin on preeclampsia model mice. Methods:Pregnant mice were injected intraperitoneally with PPAR- $\gamma$ antagonist $(2 \mathrm{mg} / \mathrm{kg} / \mathrm{d})$ at 8.5-12.5 days of pregnancy to establish PE mouse models. Two doses of LDA (10 mg/kg/d and $20 \mathrm{mg} / \mathrm{kg} / \mathrm{d})$ were given to the PE mouse models for intervention.The blood pressure, 24-hour proteinuria, urine protein/creatinine ratio, interleukin (IL) $1-\beta$ and endoglin level in peripheral blood, placental PPAR- $\gamma$ mRNA expression in placenta, the placenta weight and pathology of placenta and kidneys of mice from different groups were detected. Results:LDA effectively decreased the blood pressure increase caused by PPAR- $\gamma$ antagonist in mice, and the increase degree of urinary protein and urinary protein creatinine ratio was notably relieved.LDA also inhibited the overexpression of Endoglin and IL- $\beta$ induced by PPAR- $\gamma$ antagonist.In addition, LDA evidently increased the placental weight of preeclampsia mice.The placenta and kidney injury were obviously less serious.LDA alleviated the expression inhibition of PPAR- $\gamma$ mRNA.The remission effect of 20mgLDA was significantly better than that of 10mg.Conclusions (1) LDA has a preventive effect in PE mice induced by PPAR- $\gamma$ antagonist. (2) The preventive effect of LDA is dose-dependent in PE mouse models induced by PPAR- $\gamma$ antagonist.
\end{abstract}

Key words: Peroxisome proliferator activated receptor- $\gamma$, Low-dose aspirin, Preeclampsia, Dose-dependent

\footnotetext{
*Corresponding author : Yu Sun,Department of Obstetrics and Gynecology, Peking University First Hospital, No. 8 Sishku Street, Xicheng District, Beijing 100034, PR China
} 


\section{Background}

Preeclampsia (PE) can cause maternal multiple-organ damage and disorders and is one of the leading reasons for maternal death worldwide. The placenta is now widely considered the centre of aetiology of PE. The pathophysiological presentations of $\mathrm{PE}$ are trophoblast infiltration in myometrium, poor uterine spiral artery remodelling, placental ischaemia and hypoxia induced by insufficient placental perfusion, and endothelial cell dysfunction. The pathogenesis of PE is complicated, and vascular factors, metabolic regulation, oxidative stress, and inflammatory responses all play a role in PE. Termination of pregnancy is the most effective treatment method for PE, but this can lead to iatrogenic preterm delivery, greatly increasing the morbidity and mortality of perinatal infants. Therefore, further investigation of the pathogenesis of $\mathrm{PE}$ and seeking effective prevention and treatment routes are hot topics in the field of obstetrics.

Low-dose aspirin (LDA) is currently the preferred drug for the prevention of PE. It plays an especially important role in PE prevention in high-risk populations. In addition to the traditional cyclooxygenase (COX) pathway, non-COX-dependent pathways are also present in the prevention of PE by LDA ${ }^{[1][2]}$. Further investigating the action mechanism underlying the prevention of PE by LDA can help to better choose the applicable population.

Peroxisome proliferator-activated receptors (PPARs) are ligand-activated transcription factors that include three sub-types: PPAR- $\alpha$, PPAR- $\beta$, and PPAR- $\gamma$. PPAR $-\gamma$ is closely associated with the process of normal pregnancy. PPAR- $\gamma$ participates in the formation of the placenta during pregnancy and regulates vascular factors, the inflammatory response, and metabolic balance. Therefore, PPAR- $\gamma$ can be an important target for treating hypertension, diabetes mellitus, inflammation, and metabolic syndrome. Compared to its level in normal pregnancy, the PPAR- $\gamma$ agonist activity decreases in the peripheral blood and placenta in severe $\mathrm{PE}^{[3]}$. Fergus et al administered a PPAR $-\gamma$ specific antagonist to pregnant rats at 11-15 days of gestation, and the pregnant rats exhibited typical PE presentations ${ }^{[4]}$. Furthermore, administration of the PPAR- $\gamma$ agonist rosiglitazone to a rat PE model induced by 
reduced uterine perfusion significantly ameliorated hypertension and endothelial dysfunction and decreased the microalbumin:creatinine ratio ${ }^{[5]}$.

This study induced PE in mice by giving them a PPAR- $\gamma$ specific antagonist. Then intervention was performed through preventive administration of two different doses of aspirin to investigate the intervention effect and the action mechanism of LDA in these mice. Whether the preventive effect of LDA in mice in the PE model had a dose effect was also investigated.

Material and methods

I. Establishment of the experimental animal model and grouping

1. Experimental animals: CD-1 (ICR) mice in this study were purchased from Beijing Vital River Laboratory Animal Technology Co., Ltd., China and were housed in the Experimental Animal Centre of the Peking University First Hospital. The strain of all experimental animals was verified to conform to the requirements of international standards. The animal housing environment met the clean-grade requirement. Collection of all materials from mouse specimens in this study was approved by the Ethics Committee of the Peking University First Hospital.

The CD1 mice were all housed in an environment with a $12 \mathrm{~h} / 12 \mathrm{~h}$ day/night cycle and could access enough water and food. Female mice in oestrus showed redness, wetness and thread-like changes in the vaginal orifice. The female mice in oestrus were used for mating at around 8 weeks. Generally, mice were combined in a cage at 16:00 in the afternoon at a 3:1 ratio of female:male mice. The plug was examined in the morning on the next day, and the day of presence of the vaginal plug was counted as embryonic 0.5 day (E0.5). This study used 20 sexually matured male mice and 76 female mice. Three female mice died during model establishment.

2. Model establishment and grouping of experimental animals:

This study established the PE mouse model through intraperitoneal injection of a PPAR- $\gamma$ antagonist $(2 \mathrm{mg} / \mathrm{kg} / \mathrm{d})$ into pregnant mice at E8.5-12.5. Next, preventive intervention using different doses of LDA $(10-20 \mathrm{mg} / \mathrm{kg} / \mathrm{d})$ was performed on PE mice induced by the PPAR- $\gamma$ antagonist. 
Mice with plugs (E0.5) were randomly divided into four groups and seven sub-groups:

(1) Control group ( $\mathrm{N}=16)$ : divided into two sub-groups: a) intraperitoneal $(\mathrm{N}=8)$ and b) intragastric group $(\mathrm{N}=8)$;

(2) PPAR- $\gamma$ antagonist group (antagonist group, $\mathrm{N}=8$ );

(3) PPAR- $\gamma$ antagonist + LDA group $(\mathrm{N}=16)$ : divided into two sub-groups: a) PPAR- $\gamma$ antagonist + LDA $10 \mathrm{mg} / \mathrm{kg} / \mathrm{d}$ (antagonist + LDA $10 \mathrm{mg}, \mathrm{N}=8$ ) and b) PPAR- $\gamma$ antagonist + LDA $20 \mathrm{mg} / \mathrm{kg} / \mathrm{d}$ (antagonist + LDA $20 \mathrm{mg}, \mathrm{N}=8$ );

(4) LDA group ( $\mathrm{N}=16)$ : divided into two sub-groups: a) LDA $10 \mathrm{mg} / \mathrm{kg} / \mathrm{d}(\mathrm{N}=8)$ and b) LDA $20 \mathrm{mg} / \mathrm{kg} / \mathrm{d}(\mathrm{N}=8)$.

Pregnant mice in the PPAR- $\gamma$ antagonist group at E8.5-E12.5 were given the PPAR $-\gamma$ specific antagonist $(2 \mathrm{mg} / \mathrm{kg})$ through intraperitoneal injection every day. Mice in the PPAR $-\gamma$ antagonist + LDA group were given different doses of LDA every day starting at E0.5 through gastric gavage and the PPAR- $\gamma$ antagonist $(2 \mathrm{mg} / \mathrm{kg}$ ) every day between E8.5 and E12.5 through intraperitoneal injection. Pregnant mice in the control group were treated with normal saline at the same volume as LDA or the PPAR- $\gamma$ antagonist. Mice in the LDA group were given different doses of LDA every day starting at E0.5 through gastric gavage.

II. Sample collection

1. Measurement of the blood pressure of pregnant mice: Blood pressure was measured using a non-invasive mouse tail sphygmomanometer (Softron BP-2010A). The blood pressure measurement times were 8:00-11:00 in the morning at E0.5 and E17.5. The systolic blood pressure (SBP) was measured.

2. Measurement of 24-h urine of mice: Mice were placed in independent metabolic cages at E17.5. Mice had normal drinking water and were provided with powered mouse chow. Metabolic cages were highly sealed, and vaporization of mouse urine during testing was minimized as much as possible to meet the normal urinary output of mice in $24 \mathrm{~h}$. The urine retention devices at the bottom of the metabolic cages were removed after $24 \mathrm{~h}$. The volume was measured, and the urine was aliquoted and stored. 
3. Collection of mouse blood: After collection of mouse urine at E18.5, mice were sacrificed by cervical dislocation, and blood samples were collected immediately through the abdominal aorta. Mice were placed on a surgical frame in the supine position. The abdominal cavity was entered from the ventral midline layer by layer to fully expose the abdominal aorta. Blood was aspirated with a sterile syringe and transferred into a blood collection tube with anti-coagulation function to prevent haemolysis. The blood collection tube was centrifuged at $3000 \mathrm{rpm}$ for $10 \mathrm{~min}$ to collect the supernatant.

III. Methods

1. Detection of PPAR $-\gamma$ mRNA levels in the placenta of pregnant mice by polymerase chain reaction (PCR): Total RNA in placental tissues of pregnant mice was extracted and reverse-transcribed into cDNA. The primer sequences were PPAR- $\gamma:$ upstream 5'-AGACCACTCGCATTCCTTT-3' and downstream 5'-CACAGACTCGGCACTCAAT-3'; and GAPDH: upstream 5'-TGGTGAAGGTCGGTGTGAAC-3' and downstream 5'-GCtCCTGGaAgAtgGtGATGG-3'. The PCR cycle was $95^{\circ} \mathrm{C}$ for pre-denaturation for $30 \mathrm{~s}$ and 40 cycles of denaturation at $95^{\circ} \mathrm{C}$ for $5 \mathrm{~s}$ and annealing at $60^{\circ} \mathrm{C}$ for $30 \mathrm{~s} . \mathrm{F}=2^{-\Delta \Delta \mathrm{Ct}}$ was used to express the relative mRNA level of the target gene.

2. Measurement of the interleukin (IL)-1 $\beta$, endoglin, and creatinine levels in peripheral blood and the creatinine level in urine of pregnant mice by enzyme-linked immunosorbent assay (ELISA): The concentrations of endoglin and IL-1 $\beta$ in plasma samples of mice were measured using reagent kits from Elabscience. Urine creatinine of mice was measured using a reagent kit (DICT-500: BioAssay Systems, CA, USA).

3. Pathological section staining: Mouse kidney and placental tissues were fixed, dehydrated, cleared, embedded, and sectioned into 2- $\mu \mathrm{m}$ sections. Sections were stained with haematoxylin and eosin (HE) and periodic acid of Schiff-methenamine silver-Masson's trichrome.

IV. Statistical methods

Data are all expressed as median (minimum, maximum). Statistical analyses were 
performed using the Mann-Whitney test and the Kruskal-Wallis test. $\mathrm{P}<0.05$ indicated a difference was significant. Statistical graphs were plotted using GraphPad Prism 5.0 software.

\section{Results}

\section{Blood pressure}

After induction by the PPAR- $\gamma$ antagonist, SBP at E17.5 was significantly higher than that in the control group(Fig 1). Compared to that in the antagonist group, SBP was $21.14 \%$ lower in the antagonist + LDA $20 \mathrm{mg}$ group and $14.08 \%$ lower in the antagonist + LDA $10 \mathrm{mg}$ group, indicating that these two doses of LDA both lowered blood pressure in PE mice. After the LDA $20 \mathrm{mg} / \mathrm{kg} / \mathrm{d}$ intervention, the SBP of PE mice at E17.5 had returned mostly to normal. SBP at E17.5 was similar between the antagonist + LDA $20 \mathrm{mg}$ group and the control group, but SBP at E17.5 was significantly different between the antagonist + LDA $10 \mathrm{mg}$ group and the control group.

A

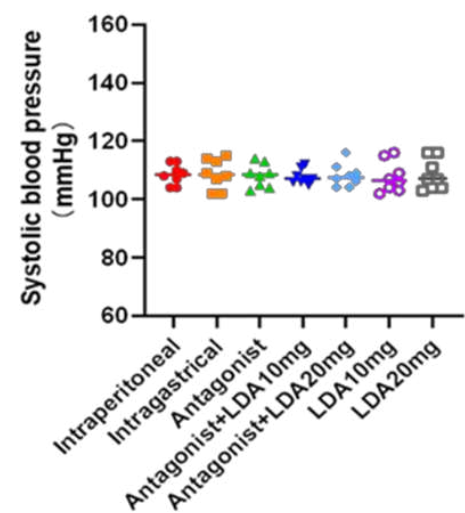

B

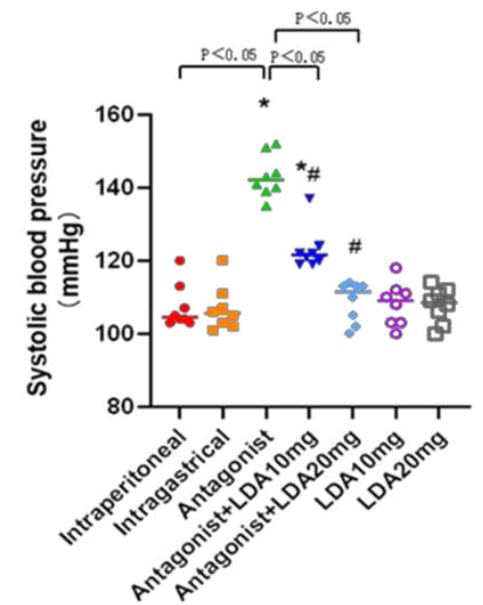

Figure 1 Systolic blood pressure of E0.5 and E17.5 in each group

A E0.5 blood pressure level of mice in different treatment groups.B E17.5 blood pressure level of mice in different treatment groups.The result is expressed as the median, the largest value and minimum value.Mann-whitney test and Kruskal-Wallis test were used for statistical analysis.*, compared with control group P\&lt;0.05.\#, $\mathrm{P}<0.05$ compared with Antagonist group.

2. 24-h urine protein and the urine protein/creatinine ratio 
The total 24-h urine protein and the urine protein/creatinine ratio were significantly higher after PPAR- $\gamma$ antagonist induction than in the control group(Fig 2). Compared to that in the antagonist group, the total 24-h urine protein of mice decreased by $51 \%$ in the antagonist + LDA $20 \mathrm{mg}$ group and $33 \%$ in the antagonist + LDA $10 \mathrm{mg} / \mathrm{kg}$ group. The urine protein/creatinine ratio of the antagonist + LDA 20 mg group decreased by $40 \%$. These differences were all significant. The urine protein/creatinine ratio of mice in the antagonist + LDA $10 \mathrm{mg}$ group was $13 \%$ lower than that in the antagonist group, but this was not a significant difference.

A

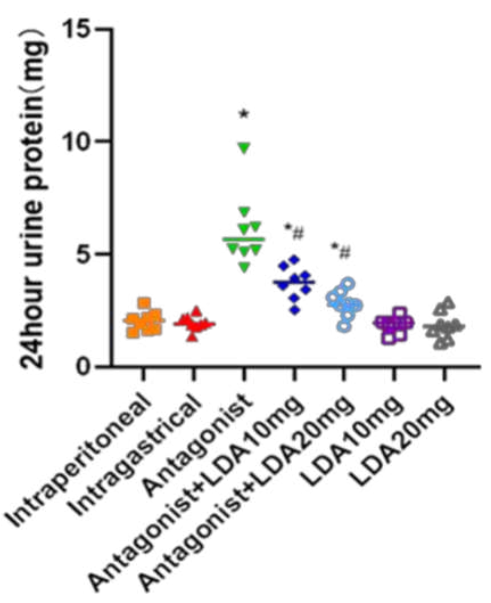

B

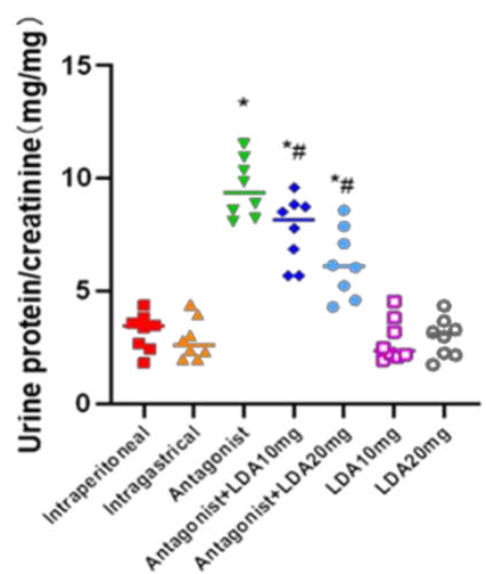

Figure 2 24-h Urine protein and urine protein creatinine ratio of E18.5 in each group

A 24-h Urine protein results of E18.5 in different treatment groups. B Urine protein/creatinine ratio of E18.5 in different treatment groups. The result is expressed as median, maximum, and minimum. Mann-whitney test and Kruskal-Wallis test were used for statistical analysis. *, and right compared with the control group, $\mathrm{P}<0.05$. \#, $\mathrm{P}<0.05$ compared with Antagonist group.

\section{Endoglin expression}

After PPAR- $\gamma$ antagonist induction, expression of peripheral endoglin was significantly higher than that in the control group(Fig 3). The endoglin concentration in peripheral blood of mice in the antagonist + LDA $10 \mathrm{mg}$ group was slightly higher than that in the antagonist group, but this difference did not have statistical significance. The endoglin concentration in peripheral blood of mice in the antagonist + LDA $20 \mathrm{mg}$ group was significantly lower than in the antagonist group. 


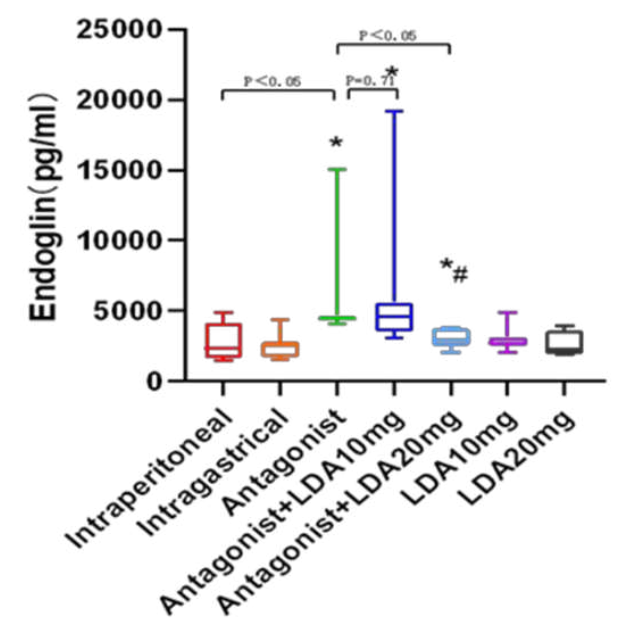

Figure3 Endoglin concentration in peripheral blood of mice in each group

The results are expressed as median, maximum, and minimum.Mann-whitney test and Kruskal-Wallis test were used for statistical analysis.*, compared with control group $\mathrm{P}<0.05$.\#, $\mathrm{P}<0.05$ compared with Antagonist group.

\section{IL-1 $\beta$ in peripheral blood}

After PPAR- $\gamma$ antagonist induction, IL-1 $\beta$ expression in peripheral blood of mice was significantly higher than that in the control group(Fig 4). Compared to that in the antagonist group, IL-1 $\beta$ in peripheral blood of mice in the antagonist + LDA 10 mg group decreased, and it decreased even further in the antagonist + LDA20 mg group. The IL-1 $\beta$ level in peripheral blood was similar between the control group and the antagonist + LDA $10 \mathrm{mg}$ group. The IL-1 $\beta$ level in peripheral blood of mice in the antagonist + LDA $20 \mathrm{mg}$ group was lower than that in the control group.

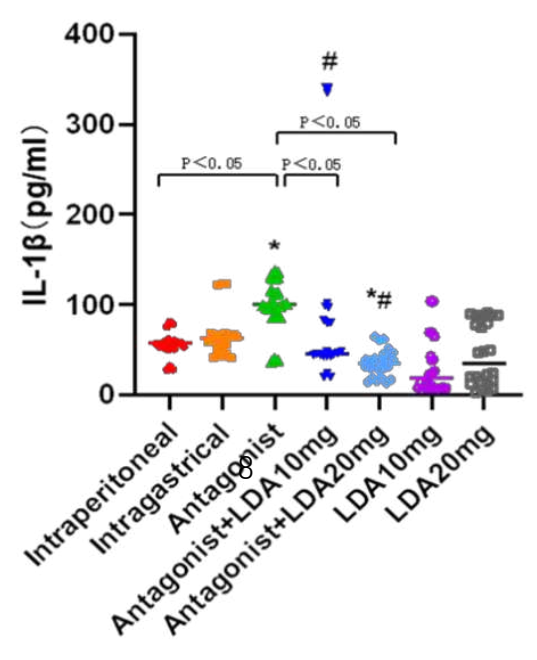


Figure 4 IL-1 concentration in peripheral blood of mice in each group

The results are expressed as median, maximum, and minimum.Mann-whitney test and Kruskal-Wallis test were used for statistical analysis.*, compared with control group $\mathrm{P}<0.05$.\#, $\mathrm{P}<0.05$ compared with Antagonist group.

5. PPAR $-\gamma$ mRNA expression in the placenta

PPAR- $\gamma$ mRNA expression in mice in the antagonist group was significantly suppressed compared to the control level(Fig 5). Compared to that in the antagonist group, the suppression of PPAR- $\gamma$ mRNA in the placenta of mice in the antagonist + LDA groups was significantly ameliorated.

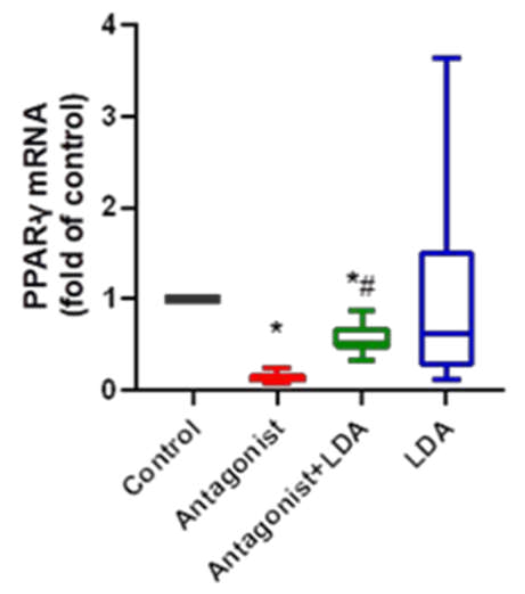

Figure 5 Expression of PPAR- mRNA in peripheral blood of mice in each group

The results are expressed as median, maximum, and minimum.Mann-whitney test and Kruskal-Wallis test were used for statistical analysis.*, compared with control group $\mathrm{P}<0.05$.\#, $\mathrm{P}<0.05$ compared with Antagonist group. 


\section{Placental weight}

The placental weight of mice in the antagonist group was $23 \%$ than it was in the control group(Fig 6).Compared to that in the antagonist group, the placental weight of mice in the antagonist + LDA $10 \mathrm{mg}$ group significantly increased, and it increased even further in the antagonist + LDA $20 \mathrm{mg}$ group. Placental weight was similar between the control group and the antagonist + LDA $20 \mathrm{mg}$ group, but it was still significantly different between the control group and the antagonist + LDA $10 \mathrm{mg}$ group. These results show that aspirin could help recover the symptoms of reduced placental weight in PE mice induced by PPAR- $\gamma$ in a dose-dependent manner. The effect of preventive administration of LDA $20 \mathrm{mg} / \mathrm{kg} / \mathrm{d}$ every day was much better.

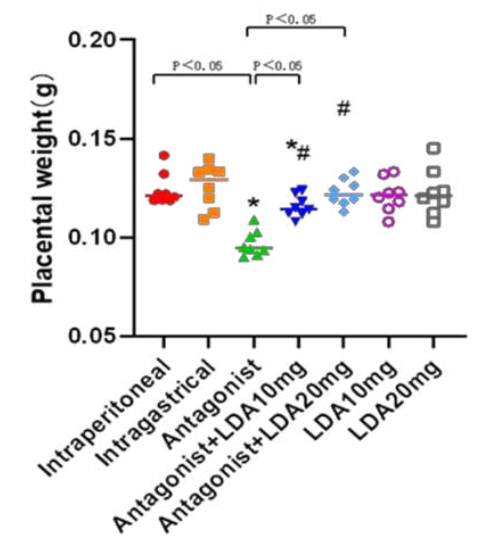

Figure 6 Placental weight of mice in each group

The results are expressed as median, maximum, and minimum.Mann-whitney test and Kruskal-Wallis test were used for statistical analysis. ${ }^{*}$, compared with control group $\mathrm{P}<0.05$.\#, $\mathrm{P}<0.05$ compared with Antagonist group.

7.Pathological results of mouse placenta

The area of placental infarction significantly decreased and the level of neutrophil infiltration improved in mice in the antagonist+LDA 10mg group compared to the antagonist group(Fig 7). The placental structure of mice in the antagonist + LDA $20 \mathrm{mg}$ group returned mostly to normal, and only a few small areas of tissue infarction could be observed on the edge of the foetal membrane. There was significantly less infarcted placental tissue and a significantly smaller infarct range 
and inflammatory exudate range in the antagonist+LDA $20 \mathrm{mg}$ group than the antagonist group. The placental pathology was similar between the LDA group and the control group.

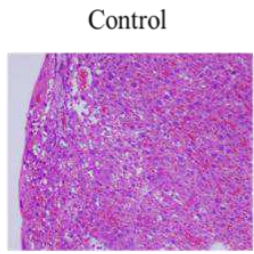

A

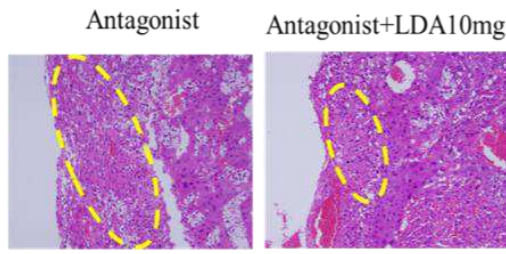

B
C

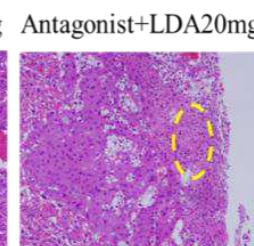

$\mathrm{D}$

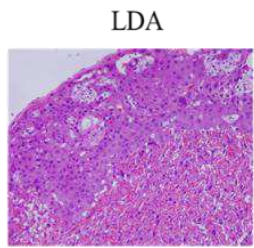

E

Figure 7 Pathological results of placenta of mice in each group $(\mathrm{HE}, \times 200)$

Figure A-E is divided into control group, Antagonist group, Antagonist+LDA10mg group, Antagonist+LDA20mg group and LDA.Pathological results of placenta of mice in group A.HE staining was used in all cases, and the magnification was 200 times.The dotted yellow line indicates inflammatory exudation with infarction parts.All pathological results randomly selected 5 groups of mice with 3 placentas in each case.

\section{Pathological results of mouse kidney}

Adding LDA $10 \mathrm{mg}$ to the antagonist ameliorated the phenomenon of glomerular basement membrane shrinkage, decreased the dilation of Bowman's capsule, and decreased renal interstitial neutrophil infiltration(Fig 8). In the antagonist+LDA 20mg group, the glomerular basement membrane did not have significant shrinkage, the Bowman's capsule dilation was not significant, and there was only local renal interstitial neutrophil infiltration. The glomerular and renal interstitial pathology was similar between the LDA group and the control group. Pathological results of renal arterioles did not have significant differences between the five groups of mice. 


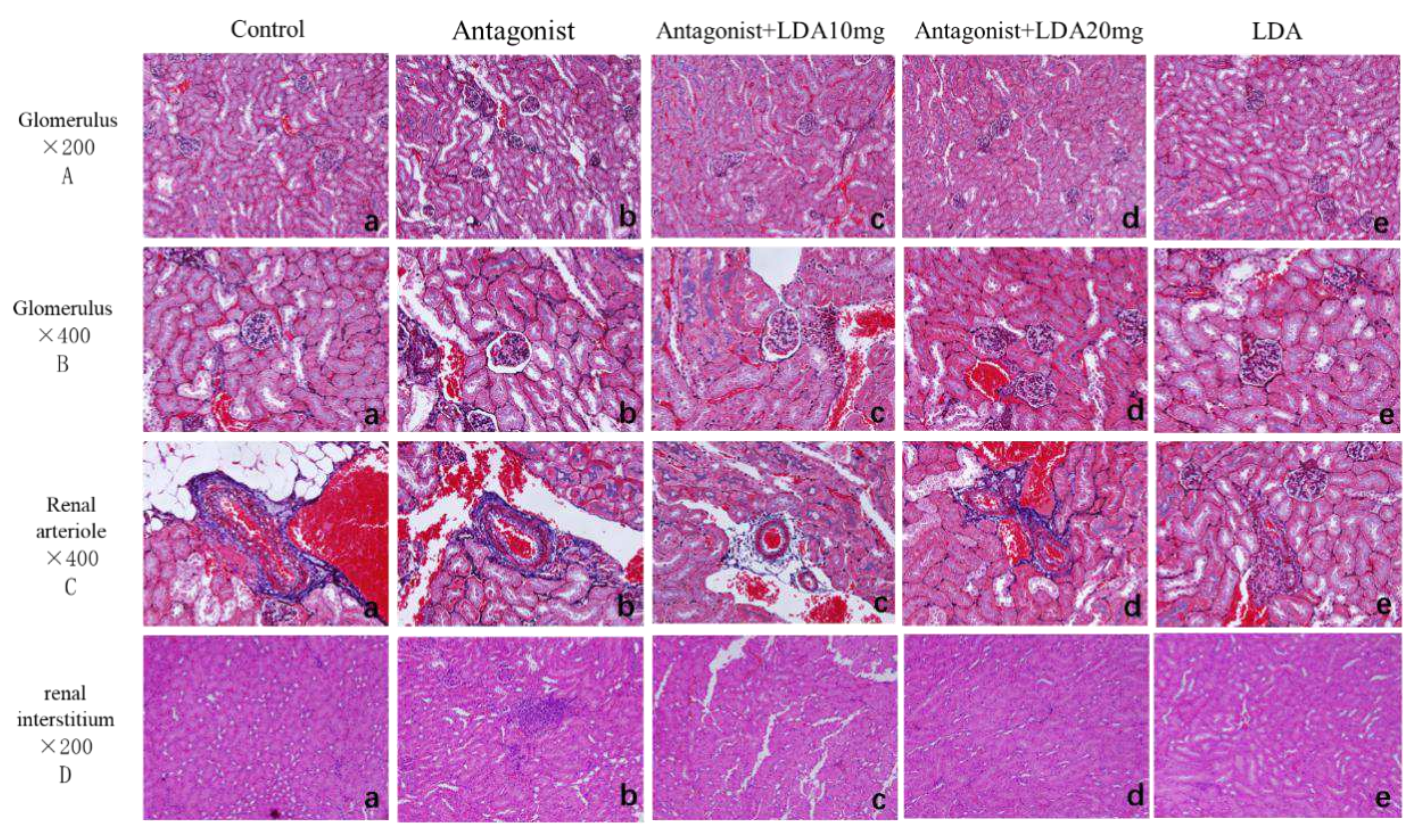

Figure 8 Renal pathological results of mice in each group $(\mathrm{HE}, \times 200)$

A(A-E) is divided into control group, Antagonist group, Antagonist+LDA10mg group, Antagonist+LDA20mg group, and LDA.The pathological results of glomerular hexamethylamine silver sheath staining with Masson staining were all 200 times magnified.B (A-E) showed small kidneys in the above five groups.The histopathological results of bulb hexylamine silver sheath staining with Masson staining were all 400 times magnified.C (A-E) was silver hexamethylamine staining of renal arterioles in five groups of mice.Pathologic results of Masson staining showed that the magnification was 400 times.D (A-E) was the results of HE staining in the renal interstitium of the five groups of mice, with all amplification multiples.For 200 times.All pathological results randomly selected 5 groups of mice with 3 cases each and 1 kidney for each case.

\section{Discussion}

1. The relationship between PPAR- $\gamma$ and PE

PPAR- $\gamma$ plays an important role in the pregnancy process. It participates in the formation of the placenta in pregnancy and regulates vascular factors, the inflammatory response, and metabolic balance ${ }^{[6]}$. This study used a PPAR $\gamma$ antagonist to successfully establish a mouse PE model. The study results indicated that PPAR- $\gamma$ participated in the pathogenesis of PE in three ways: by affecting placenta formation, inflammatory factors, and vascular factors. 
We intraperitoneally injection a PPAR- $\gamma$ specific antagonist into pregnant mice at E8.5-12.5. The results showed that the placental weight of mice significantly decreased, and the placental pathology of mice in this group had large areas of infarction combined with inflammatory exudation. These results indicated that PPAR- $\gamma$ production was suppressed, reducing the placental weight and causing necrotizing injury. Therefore, suppression of PPAR- $\gamma$ expression caused abnormal development of the placenta, eventually resulting in PE phenotypes. Kubota et al also showed that PPAR- $\gamma$ deficiency resulted in abnormal placental development ${ }^{[7]}$. Our previous in vitro study showed that a PPAR- $\gamma$ antagonist inhibited the growth of trophoblasts to slow down their growth, reduce their number, and cause cell shrinkage.

In this study, the peripheral-blood pro-inflammatory cytokine IL-1 $\beta$ was significantly higher in the PPAR- $\gamma$ antagonist group than the other groups. This result indicated that suppression of PPAR- $\gamma$ production resulted in higher levels of pro-inflammatory cytokines in peripheral blood. The lower level of PPAR- $\gamma$ could not normally regulate inflammatory responses, eventually causing PE presentations in mice.

2. Significant amelioration of phenotypes of PPAR $-\gamma$ antagonist-induced PE by LDA

This study showed that LDA intervention significantly ameliorated the hypertension in pregnant mice induced by the PPAR- $\gamma$ antagonist. In addition, LDA significantly reduced the $24-\mathrm{h}$ urine protein and the urine protein/creatinine ratio from their levels in the PPAR- $\gamma$ antagonist group. Furthermore, LDA effectively decreased the production of pro-inflammatory factors and anti-angiogenic factors and brought them to the normal pregnancy levels. Pathological results of mouse placental and kidney tissues in different treatment groups indicated that LDA significantly improved the injury to the mouse kidney and placenta induced by the PPAR- $\gamma$ antagonist. In this way, LDA effectively prevented PE in mice induced by PPAR- $\gamma$ antagonism.

3. The action mechanism of aspirin in the prevention PE in mice induced by the 
PPAR- $\gamma$ antagonist

Suppression of PPAR- $\gamma$ participated in the pathogenesis of PE through placenta formation, inflammation, and vascular effects. Preventive administration of LDA intervention significantly relieved the downregulation of PPAR- $\gamma$ mRNA in the placenta of mice induced by the PPAR- $\gamma$ antagonist. In addition, after preventive application of LDA, the over-expression of anti-angiogenic factors and inflammatory cytokines induced by the PPAR- $\gamma$ antagonist was significantly decreased, and the pathological injury to the placenta and kidney was significantly improved. In summary, the preventive application of LDA showed that the different pathways through which the PPAR- $\gamma$ antagonist promoted PE were all significantly improved. Therefore, this study suggested that LDA prevented PE through the regulation of PPAR- $\gamma$ expression.

The study of Lehmann et al showed that non-steroidal anti-inflammatory drugs (NSAIDs) can activate PPAR- $\gamma$ and PPAR- $\alpha$ during adipogenesis ${ }^{[8]}$. That study showed that NSAIDs at lower concentrations blocked COX activity to inhibit the production of adipogenic prostaglandins (PGs) but did not directly affect PPARs. However, NSAIDs at higher concentrations not only inhibited COX activity but also activated PPAR- $\gamma$ to promote adipocyte production and differentiation. Therefore, that study suggested that NSAIDs at certain concentrations can inhibit or induce adipogenesis by activating the PPAR- $\gamma$ pathway.

As one type of NSAID, aspirin, at certain concentrations, might exert biological effects by activating the PPAR- $\gamma$ pathway ${ }^{[8]}$. PPAR- $\gamma$ is a nuclear hormone receptor superfamily member that has many functions in the body. At the cell level, PPAR- $\gamma$ agonists have anti-inflammatory, anti-proliferative, anti-fibrotic, and anti-apoptotic functions. At the system level, PPAR- $\gamma$ agonists can induce haemodynamic changes in humans and animals to produce anti-hypertensive effects. PPAR- $\gamma$ might have potential relieving effects on hypertensive disorders of pregnancy ${ }^{[9]}$. McCarthy et al established a pregnant rat model of reduced uterine perfusion pressure (RUPP). RUPP rats exhibited PE-like pathological changes, and the presentations were hypertension, endothelial dysfunction, and increased microalbumin/creatinine ratio ${ }^{[10]}$. Application 
of the PPAR- $\gamma$ agonist rosiglitazone in RUPP rats improved the above symptoms to a certain degree. Thus, that study proposed that PPAR- $\gamma$ agonists might help relieve PE. In addition, that study found that PPAR- $\gamma$ agonists improved pregnancy outcomes of PE mice mainly through the activation of haeme oxygenase-1 (HO-1), which was consistent with the study of Cudmore et al, which showed that PPAR- $\gamma$ agonists reduced the production of ROS and sFlt-1 by upregulating HO-1 to reduce oxidative stress in the body of PE patients and restore the balance of angiogenic factors ${ }^{[11]}$. Other studies confirmed that activation of PPAR- $\gamma$ could restore the nitric oxide (NO) bioavailability ${ }^{[12]}$. The study of Martens et al showed that activation of PPAR- $\gamma$ promoted NO production in human aortic endothelial cells ${ }^{[13]}$.

PPAR- $\gamma$ agonists not only can interfere with oxidative stress and angiogenesis pathways but also can effectively reduce the intensity of inflammation in the body of PE patients. Hofmann et al showed that PPAR- $\gamma$ activation in monocytes and macrophages reduced the production of cytokines such as tumour necrosis factor- $\alpha$, IL-1 $\beta$, and IL-6 ${ }^{[14]}$. PPAR- $\gamma$ activation can also inhibit the pro-inflammatory effect of platelets and suppress platelet aggregation and thromboxane A2 (TXA2) release ${ }^{[15]}$. Therefore, PPAR- $\gamma$ agonists could help to restore the balance of TXA2/PGI2 in PE patients.

PPAR- $\gamma$ agonists have confirmed kidney protection functions and can effectively reduce proteinuria. Sugawara showed that PPAR- $\gamma$ agonists could reduce blood pressure, protect vascular endothelial functions, cause vasodilation of the glomerular arterioles, and reduce proteinuria production ${ }^{[16]}$. PPAR $-\gamma$ agonists in a kidney disease and glomerular hypertension model of rodents protect podocytes ${ }^{[17]}$. Especially after podocyte injury, PPAR- $\gamma$ activation plays a key protective role. Glomerular podocytes are key cells for the prevention of proteinuria, kidney failure, and cardiovascular disease.

This study performed preventive application of LDA in mice with PE induced by a PPAR- $\gamma$ antagonist, which significantly ameliorated hypertension and proteinuria symptoms. In addition, LDA effectively rescued the downregulation of PPAR- $\gamma$ mRNA. Therefore, we believe that LDA could prevent PE by regulating PPAR- $\gamma$ 
expression. PPAR- $\gamma$ mRNA expression had a wide range of values in mouse placenta in the simple LDA preventive intervention group. This result reflects the large individual differences that could confound the preventive use of LDA in clinical practice; therefore, more accurate confirmation in the LDA target population has clinical significance. Thus, whether LDA prevents PE through the activation of the PPAR- $\gamma$ pathway and its specific action mechanism still require further investigation.

\section{Aspirin intervention dose and administration time}

The preventive function of aspirin in PE mice had a dose effect. For PE mice induced by PPAR- $\gamma$ antagonists, the preventive effect of LDA at $20 \mathrm{mg} / \mathrm{kg} / \mathrm{d}$ was overall better than that at $10 \mathrm{mg} / \mathrm{kg} / \mathrm{d}$. Converting these doses for a $60-\mathrm{kg}$ adult, these result suggest that LDA at $133 \mathrm{mg} / \mathrm{d}$ would have a better effect than $66.5 \mathrm{mg} / \mathrm{d}$. In addition, this study paid close attention to the preventive effect of LDA in PE and performed LDA intervention in mice at the start of pregnancy. The results indicated that LDA $20 \mathrm{mg} / \mathrm{kg} / \mathrm{d}$ has significant preventive effects against PE induced by PPAR- $\gamma$ antagonists in mice.

Bujold et al also showed that prevention of PE by administration of LDA before 16 weeks of pregnancy was dose-dependent. Administration of aspirin $\geq 100 \mathrm{mg}$ every day significantly reduced the incidence of PE. Many observational studies also showed that daily administration of LDA $100-160 \mathrm{mg}$ might be necessary to prevent $\mathrm{PE}$, and LDA $60-80 \mathrm{mg}$ might not be enough to prevent $\mathrm{PE}^{[18]}$. The above views were all consistent with the conclusions of this study.

\section{Conclusion}

PPAR- $\gamma$ antagonists induce mice to exhibit PE phenotypes. PPAR- $\gamma$ participates in the pathogenesis of PE in three ways: regulating the formation of the placenta, inflammatory factors, and vascular factors. LDA had preventive functions in mice with PE induced by the PPAR- $\gamma$ antagonist. LDA might exert its PE-preventive function through the regulation of PPAR- $\gamma$ production. The action of LDA against PE in mice induced by the PPAR- $\gamma$ antagonist had a dose effect. 


\section{Abbreviations}

LDA: low-dose aspirin

IL: interleukin

PE: preeclampsia

COX: cyclooxygenase

PPARs: peroxisome proliferator-activated receptors

PCR: polymerase chain reaction

ELISA: enzyme-linked immunosorbent assay

HE: haematoxylin and eosin

NSAIDs: non-steroidal anti-inflammatory drugs

PGs: prostaglandins

RUPP: reduced uterine perfusion pressure

HO-1: haeme oxygenase-1

NO: nitric oxide

TXA2: thromboxane A2

\section{Declarations}

\section{Ethical approval}

The use of all data in this study was approved by the Ethics Committee of Peking University First Hospital. All methods were carried out in accordance with relevant guidelines and regulations. The procedures used in this study adhere to the tenets of the Declaration of Helsinki.

The study was carried out in compliance with the ARRIVE guidelines, according to the ARRIVE Essential 10 compliance questionnaire:

1. All the experimental and control groups are clearly identified.

2. The exact number of experimental units in each group at the start of the study is provided. The sample size chosen method is explained.

3. The criteria used for including and excluding animals, experimental units and data points are provided. There exclusions of animals experimental units and data points reported.

4. The method by which the experimental units were allocated to control and 
treatment groups is described.

5. The researchers were aware of the group allocation at any stage of the experimental and data analysis.

6. For all experimental outcomes presented, details of what parameter was measured are provided exactly.

7. The statistical approach used to analyse each outcome is detailed. There is description of methods used to assess whether data met statistical assumptions.

8. All species of animal are used specified. The sex of the animals is specified. At least one of age, weight or developmental stage of the animals specified.

9. Both the timing and frequency with which procedures took place are specified. Details of acclimatisation periods to experimental locations are provided.

10.Descriptive statistics for each experimental group are provided, with a measure of variability. The effect size and confidence interval is provided.

\section{Consent for publication}

Not Applicable.

\section{Availability of data and materials}

The datasets obtained and/or analyzed during the current study are available. From the corresponding author on reasonable request.

\section{Competing interests}

The authors declare that they have no competing interests.

\section{Author contributions}

YBG and YCZ designed the research and drafted the manuscript.YS and HXY were chief director of maternal and fetal unit, and participted in its design and coordination. All authors have accepted responsibility for the entire content of this manuscript and approved its submission. 


\section{Funding}

1.National Key R\&D Program of China "Research on prevention and control of reproductive health and major birth defects: regulation of human embryo implantation and related mechanisms of major pregnancy diseases"(2017YFC1001404).

2. National natural science foundation of China"The mechanism of low dose asprin regulating soluble endoglin expression to prevent preeclampsia"(81701466).

3. Major projects of the national natural science foundation of China"Sample library constrution and plasma biomarker investigation on recurrent spontaneous abortion and preeclampsia"(81490745).

\section{Acknowledgements}

We are grateful for the funding.

\section{Reference}

[1] Doridot L , Passet B , Mehats C , et al. Preeclampsia-Like Symptoms Induced in Mice by Fetoplacental Expression of STOX1 Are Reversed by Aspirin Treatment. Hypertension 61(3),662-668(2013).

[2] Erdemli H K, Yıldırımlar P , Alper T Y , et al. Increased serum heme oxygenase-1 levels as a diagnostic marker of oxidative stress in preeclampsia. Hypertension in Pregnancy 33(4),488-497(2014).

[3] Waite, Leslie L, Louie, Rachel E, Taylor, Robert N. Circulating Activators of Peroxisome Proliferator-Activated Receptors Are Reduced in Preeclamptic Pregnancy. Journal of Clinical Endocrinology \& Metabolism 90(2),620-626(2005).

[4] Fergus P. McCarthy, Sascha Drewlo, Fred A. English, et al.Evidence Implicating Peroxisome Proliferator-Activated Receptor- $\gamma$ in the Pathogenesis of Preeclampsia. Hypertension 58(5),882-887(2011).

[5] Fergus P. McCarthy, Sascha Drewlo, John Kingdom, et al. Peroxisome 
Proliferator-Activated Receptor- $\gamma$ as a Potential Therapeutic Target in the Treatment of Preeclampsia. Hypertension 58,280-286(2011).

[6] T.Yee Khong. Placental vascular development and neonatal outcome. Semin Neonatol 9(4):255-263(2004).

[7] Kubota N, Terauchi Y, Miki H, Tamemoto H, Yamauchi T,Komeda K et al. (1999). PPAR gamma mediates high-fat diet-induced adipocyte hypertrophy and insulin resistance.Mol Cell 4(4), 597-609(1999).

[8] Lehmann J M , Lenhard J M , Oliver B B , et al. Peroxisome Proliferator-activated Receptors $\gamma$ and $\alpha$ Are Activated by Indomethacin and Other Non-steroidal Anti-inflammatory Drugs. Journal of Biological Chemistry 272(6),3406-3410(1997).

[9] Usuda D, Kanda T. Peroxisome proliferator-activated receptors for hypertension. World J Cardiol 6(8),744-754(2014).

[10] Mccarthy F P , Delany A C , Kenny L C , et al. PPAR- $\gamma$ a possible drug target for complicated pregnancies. British Journal of Pharmacology 168(5),1074-85,(2013).

[11] Cudmore M , Ahmad S , Al-Ani B , et al. Negative Regulation of Soluble Flt-1 and Soluble Endoglin Release by Heme Oxygenase-1. Circulation 115(13),1789-1797(2007).

[12] Sorrentino SABahlmann. Oxidant stress impairs in vivo reendothelialization capacity of endothelial progenitor cells from patients with type 2 diabetes mellitus:Restoration by the peroxisome proliferator-activated receptor- $\gamma$ agonist rosiglitazone. Digest of the World Core Medical Journals 116(2),163-173(2007).

[13] Martens D F M A C , Visseren F L J , Lemay J , et al. Metabolic and Additional Vascular Effects of Thiazolidinediones. Drugs 62(10),1463-1480(2002).

[14] Hofmann C, Lorenz K , Braithwaite S S , et al. Altered gene expression for tumor necrosis factor-alpha and its receptors during drug and dietary modulation of insulin resistance. Endocrinology 134(1),264-270(1994).

[15] Akbiyik F , Ray D M , Gettings K F , et al. Human bone marrow megakaryocytes and platelets express PPAR, and PPAR agonists blunt platelet release of CD40 ligand and thromboxanes. Blood 104(5),1361-1368(2004).

[16]Sugawara A, Uruno A, Kudo M, et al. PPAR $\gamma$ Agonist Beyond Glucose Lowering Effect. The Korean Journal of Internal Medicine 26(1),19-24(2011). 
[17] Miceli I, Burt D, Tarabra E , et al. Stretch reduces nephrin expression via an angiotensin II-AT(1)-dependent mechanism in human podocytes: effect of rosiglitazone. Am J Physiol Renal Physiol 298(2),F381(2010).

[18] Roberge S, Nicolaides K, Dermers S, et al. The role of aspirin dose on the prevention of preeclampsia and fetal growth restriction: systematic review and meta-analysis. Am J Obstet Gynecol 216(2),110-120(2017). 